\title{
Calibrations of Amber Perspex-PMMA Dosimeter in the CDTN Gamma Irradiator Operational Conditions
}

\author{
Luiz Carlos Duarte Ladeira, Amir Zacarias Mesquita and Márcio Tadeu Pereira \\ Nuclear Technology Development Center-CDTN, National Nuclear Energy Commission-CNEN, Belo Horizonte 31270-901, \\ Brazil
}

Received: December 18, 2014 / Accepted: February 02, 2015 / Published: March 31, 2015.

\begin{abstract}
This paper presents results of PMMA (polymethylmethacrylate) dosimeters calibrations that are used as routine dosimeters in CDTN (Nuclear Technology Development Center) Gamma Irradiation Laboratory. The study was carried out within the framework of the Facility Quality Assurance Program with the purpose of to determine the calibration curve and accuracy of the Harwell Amber Perspex $3042 \mathrm{~W}$ dosimeters. The irradiations of the dosimeters were made in operational conditions of a Gamma Irradiator at CDTN, an R \& D (research and development) institute connected to the CNEN (National Nuclear Energy Commission) (Brazil). The values for estimate of the expanded uncertainties achieved are typical of a routine dosimetry system.
\end{abstract}

Key words: Accuracy, PMMA dosimeter, gamma processing, absorbed dose, irradiator.

\section{Introduction}

Any application that uses ionizing radiation, in order to achieve specific biological, chemical or physical effects is classified in a general way as an irradiation process. In such process, the material is intentionally irradiated, during a certain time interval to maintain, modify or improve its characteristics. In this time interval, the product absorbs only a fraction of the energy carried by the radiation that strikes it. This fraction, which is called radiation absorbed dose, depends on the mass of the product, its composition and the exposure time. The dose unit is the Gy (gray), which is defined as the absorption of 1 joule of energy per $\mathrm{kg}$ of product.

In order to determine the absorbed dose, irradiation facilities have used different measurement systems that characterize a dosimetry system. Dosimetry represents an important function in radiation processing, where large absorbed doses and dose rates from photon and electron sources have to be measred

Corresponding author: Luiz Carlos Duarte Ladeira, researcher, research fields: irradiators and gamma processing. E-mail: lcdl@cdtn.br. with reasonable accuracy.

Proven dosimetry systems are widely used to perform radiation measurements in development of new processes, validation, qualification and verification (quality control) of established processes and archival documentation of day-to-day and plant-to-plant processing uniformity. Proper calibration and traceability of routine dosimetry systems to standards are crucial to the success of many large-volume radiation processes.

It is essential that, users perform their own separate calibration for their own instrumentation and conditions of use [1].

This paper presents the calibrations results of Amber Perspex 3042 W PMMA (polymethylmethacrylate) dosimeters, produced by Harwell that are used as routine dosimeters in the CDTN (Nuclear Technology Development Center) Gamma Irradiation Facility [1]. The study was carried out within the framework of the Facility Quality Assurance Program with the purpose of to determine the calibration curve and uncertainties of the dosimeter in the facility operational conditions. 


\section{Experiments}

\subsection{Dosimetry System}

The following components of PMMA routine dosimetry systems are used in CDTN Gamma Irradiation Laboratory.

\subsubsection{PMMA Dosimeter}

The dosimeters studied are named Amber Perspex (4034, batch $\mathrm{W}$ ), that cover the range which from 1 $\mathrm{kGy}$ to $30 \mathrm{kGy}$. They are presented in the form of small rectangular pieces (Fig. 1), optically transparent and individually sealed in aluminum sachets.

The pieces are kept in their protective cases during and after irradiation, only being open at the time of their reading. This procedure is justified by the need to preserve the radiosensitive material to dirt, abrasion and environmental conditions (light and humidity) factors that may influence the dosimeter quality. When exposed to radiation, they exhibit a change in its specific absorbance, which is characterized as a function of absorbed dose. This absorbance change is measured by UV-Vis (ultraviolet-visible) spectrophotometry.

\subsubsection{Spectrophotometer}

For the measurement of the dosimeters optical absorbance, the spectrophotometer shown in Fig. 2 was used. It was manufactured by Micronal and its model is B572 [2]. Its main characteristics are shown in Table 1.

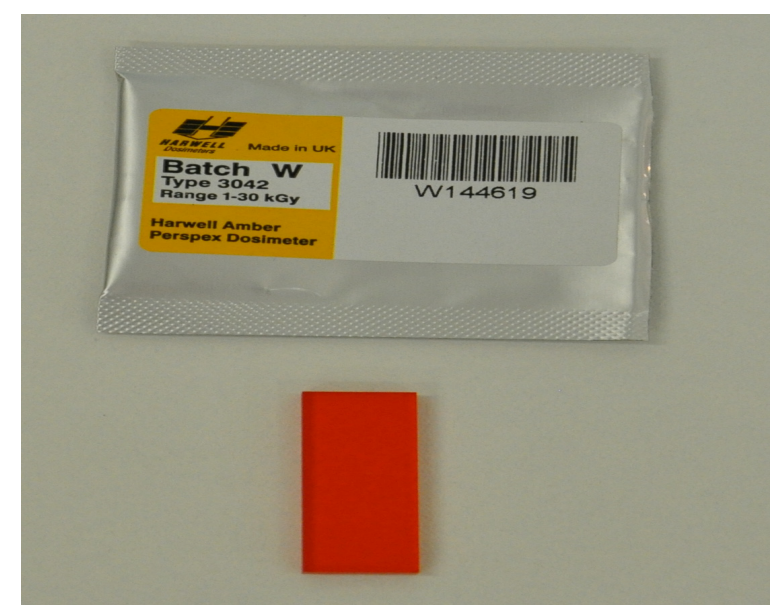

Fig. 1 Harwell Amber Perspex 4034.

\subsubsection{Thicknessgauge}

For the dosimeters thickness measurements, a calibrated electronic outside micrometer shown in Fig. 3 was used. It was manufactured by digimess instruments, has a measurement range of 0-25 $\mathrm{mm}$ and accuracy of $\pm 0.002 \mathrm{~mm}$ [3].

\subsection{The Irradiation Facility}

The CDTN irradiator is a GB-127 (gamma beam-127) type, manufactured by Nordion [4]. The GB-127 is a dry storage gamma panoramic irradiator that uses Nordion's F-127 shipping container for source storage and operation. Products to be irradiated are placed inside the biological shield, either on turntables or directly on the floor. The source is raised by a

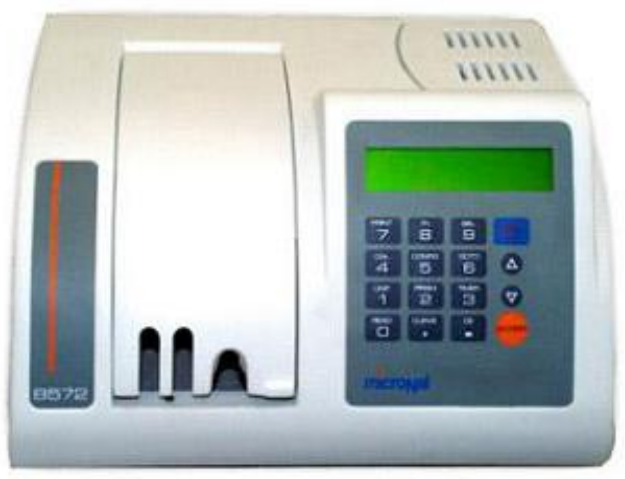

Fig. 2 Spectrophotometer UV-Vis.

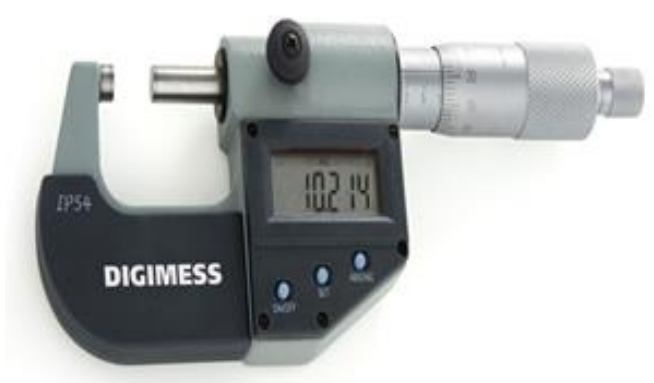

Fig. 3 Electronic outside micrometer.

Table 1 Spectrophotometer characteristics.

\begin{tabular}{ll}
\hline Wavelength range & $340 \mathrm{~nm}$ to $1,000 \mathrm{~nm}$ \\
\hline Bandwidth & $10 \mathrm{~nm}$ \\
Wavelength accuracy & $\pm 2 \mathrm{~nm}$ \\
Absorbance band & -0.300 to 3.000 \\
Absorbance accuracy $^{*}$ & \pm 0.012 \\
\hline
\end{tabular}

${ }^{*}$ Verified through a set of standards filter. 
Table 2 Irradiator specifications.

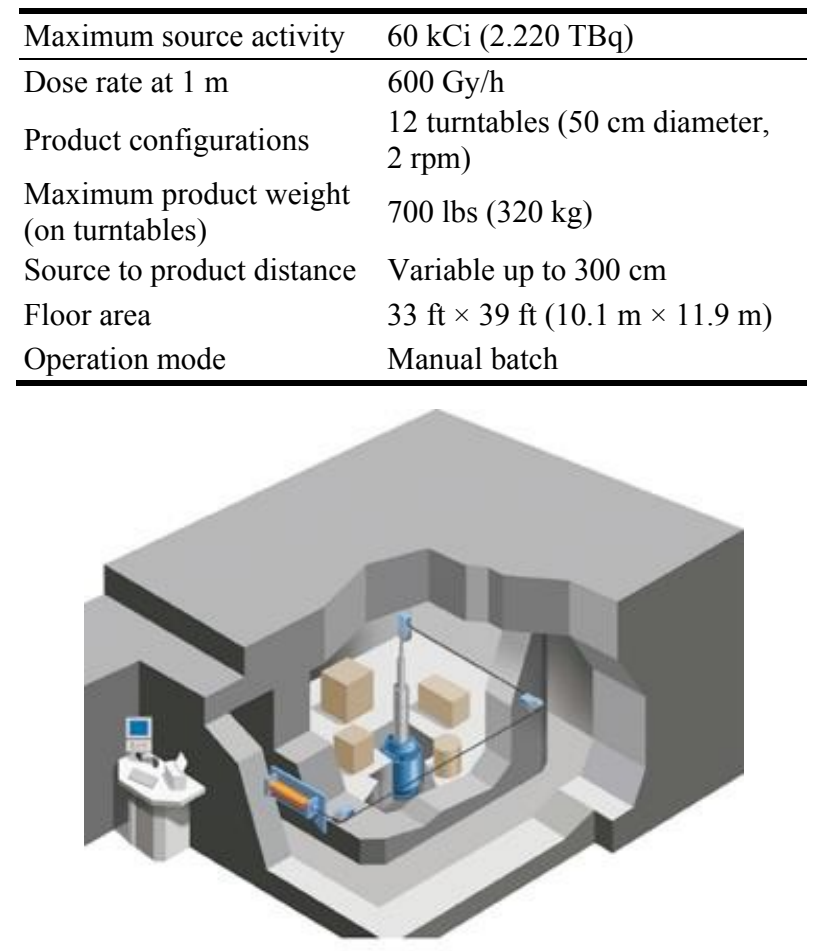

Fig. 4 Irradiator cut away view.

pneumatic hoist. Table 2 shows the irradiator specifications and Fig. 4 presents a cut away view of the irradiation facility.

\subsection{Dosimeters Positioning}

The experimental setup for calibration consisted of a PVC (polyvinylchloride) pipe divided into 11 irradiation points with three dosimeters per point. The wood holder shown in Fig. 5 was fabricated for the dosimeters positioning.

The assembly was placed in the irradiation room, with the horizontal dosimeters midline coinciding with the source center plane, as shown in Fig. 6.

\section{Methodology}

\subsection{Absorbed Doses}

The dosimeters were calibrated according to ISO (International Organization for Standardization)/ASTM (American Society for Testing and Materials) standard [5]. Establishing the calibration curve was used as reference Fricke dosimeter, which is the secondary

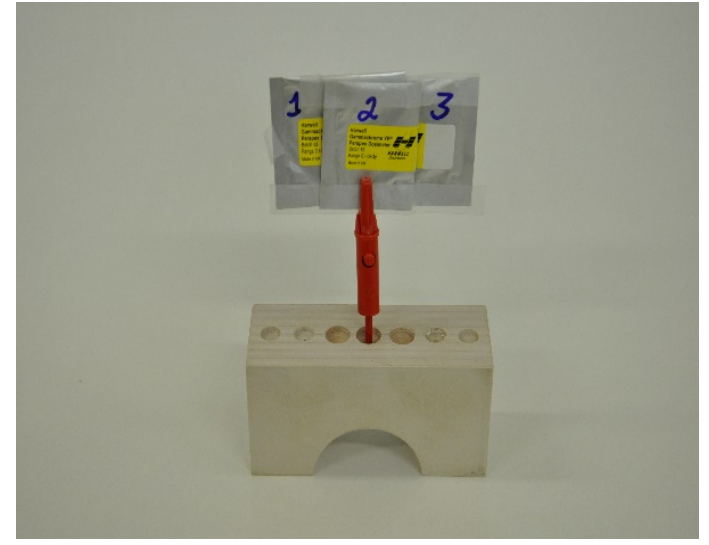

Fig. 5 Support with three dosimeters.

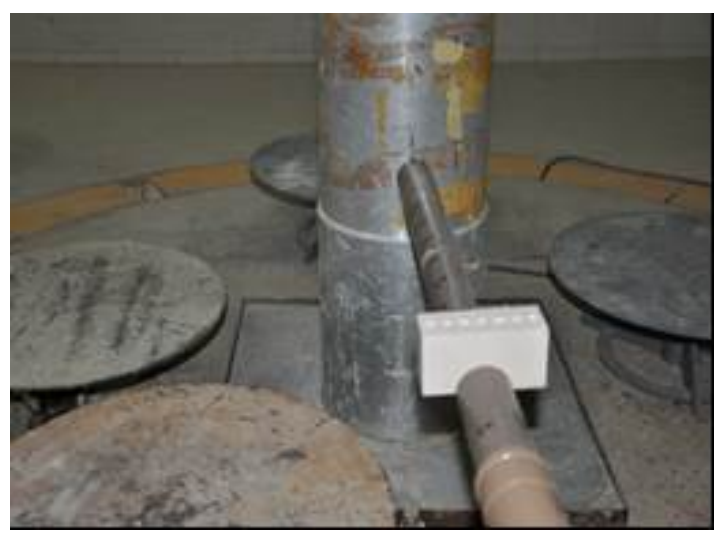

Fig. 6 Dosimeters positioning.

standard used in the CDTN Gamma Irradiation Laboratory. This dosimeter was calibrated against a primary standard [6].

The dosimeters irradiations were carried out under conditions similar to those in routine use, according manufacture's recommendations [1].

All influence quantities (e.g., shelf-life, temperature, relative humidity, among others) associated with pre-irradiation, irradiation and post-irradiation conditions were taken into account [5].

The dosimeters optical absorbance was measured with the spectrophotometer at wavelength $\lambda=603 \mathrm{~nm}$ (1-10 kGy) and $\lambda=651 \mathrm{~nm}$ (10-30 kGy).

For PMMA dosimeter, the specific absorbance $A_{s p}$ is the dosimeter response [5]:

$$
A_{s p}=\left(A_{\lambda} / d\right)
$$

where, $A_{\lambda}=$ optical absorbance at a selected wavelength $(\lambda) ; d=$ optical path length. 


\subsection{Uncertainties}

The result of a measurement is only an estimate of the measurement value. Thus, this result is only complete when accompanied by an uncertainty indication of the estimate.

Uncertainty is defined as the associated measure value with that characterizes the distribution of values that could reasonably be attributed to the measuring parameter. On the other hand, the error is defined as the difference between a measurement result and the actual quantity value.

In order to obtain a significant value of the measurement of the absorbed dose, the uncertainties associated with its measurement must be estimated and their magnitudes quantified.

Uncertainties can be classified according to the type of effect that causes (random or not random) or based on their evaluations method (Type A or Type B). The random type comes from unpredictable variations (stochastic) parameters of influence. It can be reduced by increasing the number of observations but not eliminated [7]. Furthermore, the systematic error arises from the known influence parameters effects. It will be reduced if the effect can be quantified.

The purpose of classification in Types A and B indicates the two different ways of evaluating uncertainty components.

In Type $\mathrm{A}$, the evaluation method is based on statistical analysis of a series of observations, thus, the uncertainty is obtained from a probability density function derived from a frequency distribution observed. In the Type B, the evaluation method is based on measuring instruments information, calibration, certificates, chemical purity, etc. The uncertainty in this case is obtained from a density of pre-defined probability.

The uncertainties sources in dosimetry usually reside in factors such as the dose absorbed by the dosimeter during calibration of the system, the analysis of the response of the dosimeter, the adjustment of calibration curves for the dosimetric data, among others [8].

Each uncertainty source usually consists of several components of both Types A and B.

For sources of independent (uncorrelated) uncertainty, the combined uncertainty is obtained by combining all of the standard uncertainties-Types A and $\mathrm{B}$. This combined standard uncertainty is defined by:

$$
U_{C}=\sqrt{U_{A}^{2}+U_{B}^{2}}
$$

For related uncertainty sources, the effects of these correlations should be taken into account in determining the combined standard uncertainty.

The expanded uncertainty is obtained by multiplying the combined standard uncertainty by a coverage factor $k$ :

$$
U=k U_{C}
$$

For normal probability distributions if $U_{A} \leq U_{B}$, then $k=1$ corresponds to a $66 \%$ confidence level, $k=2$ corresponds to $95 \%$ and $k=3$ corresponds to $99 \%$.

The uncertainty for $n$ points is obtained by calculating the expanded variance $V$ :

$$
V=(1 / n) \sum_{i=1}^{n}\left(U_{C}^{2}\right)_{i}
$$

And the global combined standard uncertainty $U_{G C}$ is obtained by:

$$
U_{G C}=\sqrt{V}
$$

\section{Results and Discussion}

\subsection{Dosimeters Irradiations Results}

Table 3 shows the results of dosimeters irradiations which were performed on December 19, 2013. The changes in absorbance of the dosimeters were determined at a wavelength of $\lambda=603 \mathrm{~nm}$.

The means specific absorbance $\overline{\left(A_{s p}\right)}$ considers the measurement of the three dosimeters positioned at each point.

Table 4 shows the results of the dosimeters irradiation whose absorbance was determined at a wavelength $\lambda=651 \mathrm{~nm}$. 
Table 3 Results at $\lambda=603 \mathrm{~nm}$.

\begin{tabular}{llll}
\hline Point & $\begin{array}{l}\text { Distance } \\
(\mathrm{m})\end{array}$ & $\begin{array}{l}\overline{A_{s p}} \\
\left(\mathrm{~mm}^{-1}\right)\end{array}$ & $\begin{array}{l}\text { Dose } \\
(\mathrm{kGy})\end{array}$ \\
\hline 1 & 0.1 & 0.475 & 16.70 \\
2 & 0.15 & 0.351 & 11.55 \\
3 & 0.20 & 0.263 & 8.35 \\
4 & 0.25 & 0.190 & 6.30 \\
5 & 0.30 & 0.161 & 4.92 \\
6 & 0.35 & 0.130 & 3.95 \\
7 & 0.40 & 0.106 & 3.24 \\
8 & 0.45 & 0.087 & 2.70 \\
9 & 0.50 & 0.072 & 2.29 \\
10 & 0.60 & 0.053 & 1.70 \\
11 & 0.80 & 0.030 & 1.04 \\
\hline
\end{tabular}

Table 4 Results at $\lambda=651 \mathrm{~nm}$.

\begin{tabular}{llll}
\hline Point & $\begin{array}{l}\text { Distance } \\
(\mathrm{m})\end{array}$ & $\begin{array}{l}\overline{A_{s p}} \\
\left(\mathrm{~mm}^{-1}\right)\end{array}$ & $\begin{array}{l}\text { Dose } \\
(\mathrm{kGy})\end{array}$ \\
\hline 1 & 0.1 & 0.013 & 1.04 \\
2 & 0.15 & 0.025 & 1.70 \\
3 & 0.20 & 0.035 & 2.29 \\
4 & 0.25 & 0.042 & 2.70 \\
5 & 0.30 & 0.052 & 3.24 \\
6 & 0.35 & 0.063 & 3.95 \\
7 & 0.40 & 0.079 & 4.92 \\
8 & 0.45 & 0.130 & 8.35 \\
9 & 0.50 & 0.172 & 11.55 \\
10 & 0.60 & 0.230 & 16.70 \\
11 & 0.80 & 0.343 & 29.66 \\
\hline
\end{tabular}

The mean specific absorbance $\overline{\left(A_{s p}\right)}$ considers, also the measurement of the three dosimeters positioned at each point.

\subsection{Uncertainties Evaluation}

The uncertainties sources identified in measurements were the dose determination (Fricke reference dosimeter), the source decay, the dosimeter thickness (optical path length) measuring, the spectrophotometer accuracy and the dosimeter repeatability.

The dose determination uncertainty found was $\pm 2.48 \%$. The source decay uncertainty has a value of $\pm 0.01 \%$ while the dosimeter thicknesses are \pm 0.002 $\mathrm{mm}$ and \pm 0.012 for spectrophotometer reading.

The dosimeter repeatability is found by calculating the average standard deviation between absorbance measurements at each point. Table 5 shows, as an example, the calculations for the uncertainty in the absorbed dose at point \#1.

The same calculation was performed for all calibration points, in order to obtain the expanded variance, whose value is:

$$
V=(1 / 9) \sum_{i=1}^{11}\left(U_{C}^{2}\right)_{i}
$$

Thus, the overall combined standard uncertainty is obtained by:

$$
U_{G C}=\sqrt{V}
$$

With this methodology, the values $3.34 \%$ (range 0-17 kGy) and 3.46\% (range 0-30 kGy) for the overall combined standard uncertainties were found.

\subsection{Calibration Curves}

The relation between the dosimeter response and the absorbed dose is represented by the fourth degree adjusted polynomial, in the range of 0-17 kGy.

For this range, the calibration curve is:

$$
A_{s p}=\mathrm{a}+\mathrm{b} D+\mathrm{c} D^{2}+\mathrm{d} D^{3}+\mathrm{e} D^{4}
$$

where, $A_{s p}\left(\mathrm{~mm}^{-1}\right)=$ specific absorbance; $D(\mathrm{kGy})=$ absorbed dose; $\quad \mathrm{a}=-8.57724 \mathrm{E}-03 ; \quad \mathrm{d}=$ 7.010120E-06; $\quad \mathrm{b}=3.71476 \mathrm{E}-02$; $\mathrm{e}=-2.49855 \mathrm{E}-08 ; \mathrm{c}=-6.00360 \mathrm{E}-04$.

The Fig. 7 shows the calibration curve. Considering the curve fit factor, which is $0.93 \%$, and the overall combined standard uncertainty of $3.34 \%$, is obtained a value of $3.47 \%$ for the combined standard uncertainty, when using Eq. (8) for specific absorbance determinations.

Specific absorbance determinations with Eq. (8) are affected with an expanded uncertainty of $\pm 6.94 \%$ at the $95 \%$ confidence level.

\subsubsection{Range $0 \mathrm{kGy}$ to $30 \mathrm{kGy}$}

For this range, the calibration curve is:

$$
A_{s p}=\mathrm{a}+\mathrm{b} D+\mathrm{c} D^{2}+\mathrm{d} D^{3}+\mathrm{e} D^{4}
$$
where, $A_{s p}\left(\mathrm{~mm}^{-1}\right)=$ specific absorbance; $D(\mathrm{kGy})=$ dose rate; $\mathrm{a}=-5.89497 \mathrm{E}-03 ; \mathrm{d}=2.38222 \mathrm{E}-07$; $\mathrm{b}=1.87171 \mathrm{E}-02 ; \quad \mathrm{e}=6.23733 \mathrm{E}-08 ; \quad \mathrm{c}=$ $-2.96574 \mathrm{E}-04$. 
Table 5 Uncertainty calculation for the irradiation point number \#1 $(\lambda=603 \mathrm{~nm})$.

\begin{tabular}{|c|c|c|c|c|c|}
\hline \multicolumn{3}{|l|}{ Calibration } & \multicolumn{3}{|l|}{ Uncertainty } \\
\hline \multirow{2}{*}{ Dose (kGy) } & \multirow{2}{*}{ Dosimeter } & \multirow{2}{*}{$A_{s p}\left(\mathrm{~mm}^{-1}\right)$} & \multirow{2}{*}{ Uncertainty sources } & \multicolumn{2}{|c|}{ Type } \\
\hline & & & & $\mathrm{A}(\%)$ & B (\%) \\
\hline \multirow{3}{*}{16.70} & 1 & 0.473 & Dose determination & & 2.48 \\
\hline & 2 & 0.477 & Source decay & & 0.01 \\
\hline & 3 & 0.474 & Dosimeter thickness & & 0.03 \\
\hline \multirow{2}{*}{\multicolumn{2}{|c|}{ Mean specific absorbance }} & \multirow{2}{*}{0.475} & Spectrophotometer reading & & 1.4 \\
\hline & & & Repeatability & 0.25 & \\
\hline \multirow{3}{*}{\multicolumn{2}{|c|}{ Uncertainty $(95.45 \%)$}} & \multirow{3}{*}{0.027} & Combined separated uncertainty & 0.25 & 2.85 \\
\hline & & & Combined standard uncertainty & 2.86 & \\
\hline & & & Relative expanded uncertainty $(95.45 \%)$ & 5.72 & \\
\hline
\end{tabular}

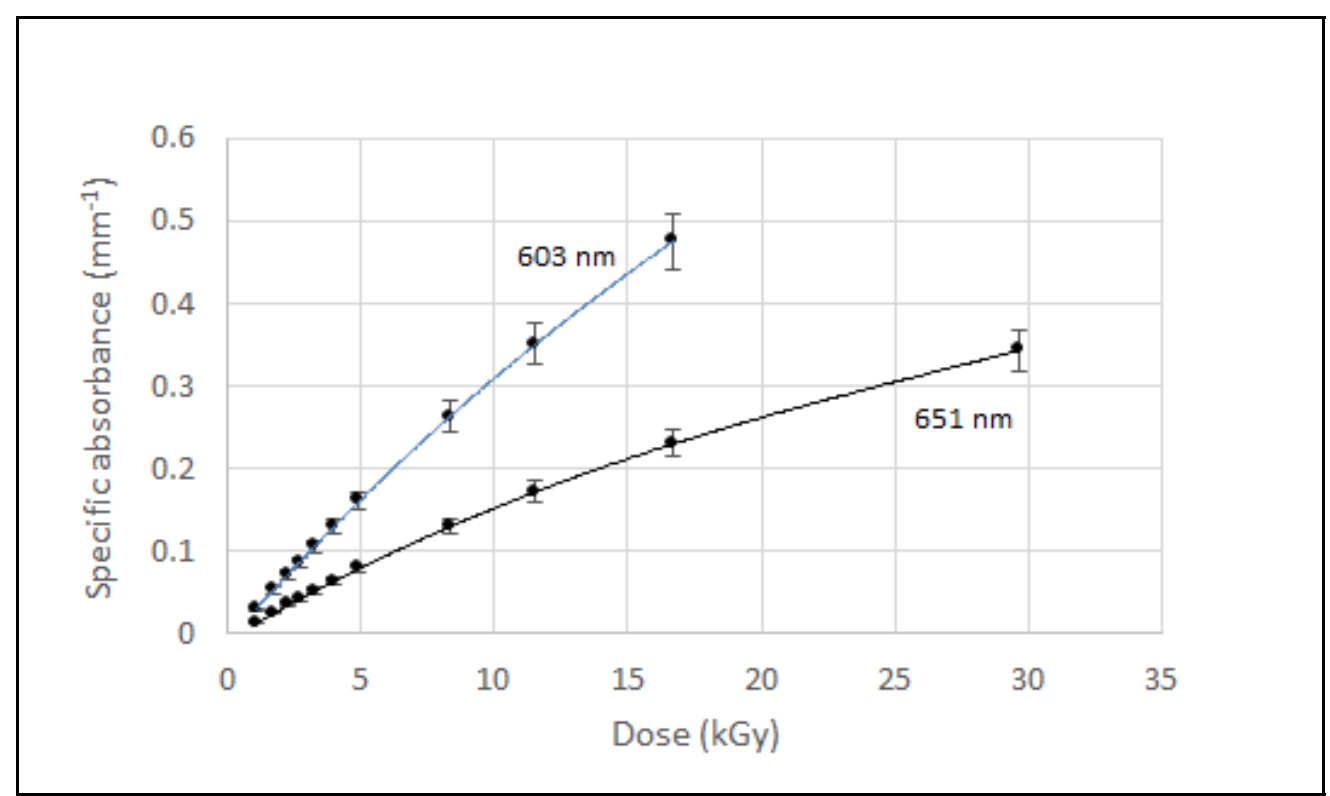

Fig. 7 Calibration curves.

The Fig. 7 shows the calibration curve. Considering the curve fit factor, which is $0.78 \%$, and the overall combined standard uncertainty of $3.46 \%$, is obtained a value of $3.55 \%$ for the combined standard uncertainty, when using Eq. (9) for specific absorbance determinations.

Specific absorbance determinations with Eq. (9) are affected with an expanded uncertainty of $\pm 7.10 \%$ at the $95 \%$ confidence level.

\section{Conclusions}

The values of $\pm 6.94 \%$ and $7.10 \%$ for estimate of the expanded uncertainties achieved with the carried out measurements are typical of a routine dosimetry system, that is of the order of $\pm 6 \%(k=2)[5]$.

This result makes clear that the use of PMMA Amber Perspex dosimeters in the routine dosimetry of the CDTN's Gamma Irradiation Laboratory is appropriate and contributes to the facility's irradiation processes quality assurance. That is true because a good routine dosimetry contributes to obtain reliable results in irradiation protocols.

\section{Acknowledgments}

The authors wish to thank Temóteo, A. J. and Felix, O. C. from the operation staff of Gamma Irradiation Laboratory of CDTN.

The research support foundation of the state of 
Minas Gerais and the Brazilian Council for Scientific and Technological Development supports this research.

\section{References}

[1] HARWELL. 1999. Dosimetry Systems for radiation Processing. Oxfordshire: Harwell Dosimeters Limited.

[2] MICRONAL. 2002. Instructions for Use of Spectrophotometer B572. São Paulo: MICRONAL S/A, 2002, IU-B572.

[3] DIGIMESS. 2012. Instructions for Use of the Electronic outside Micrometer 0-25 $\mathrm{mm}$. São Paulo: Digimess Instrumentos de Precisão Ltda..

[4] MDS NORDION. 2001. Licensing Information Package for Gamma Irradiator GB-127 Serial Number IR-214.
Québec: S.N., 2001. IN/LP 1725 IR214(1).

[5] ISO/ASTM. 2012. Practice for Use of a Polymethylmethacrylate Dosimetry System. Switzerland: ISO (International Organization for Standardization), ISO/ASTM 51276-2002.

[6] Conceiçao, C. C. S. 2006. Implementation of Chemical Dosimetry of High Dose Rates for Industrial Facilities. Rio de Janeiro: COPPE.

[7] ISO. 1995. ISOGUM95-Guide to the Expression of Uncertainty in Measurements. Switzerland: International Organization for Standardization, ISBN 92-67-10188-9.

[8] Conceiçao, C. C. S., Tauata, L. J., and Edgar, F. O. J. 2006. "Implementation of Methodology for High Dose Dosimetry." IMEKO (Inernational Measurement Confederation). Accessed August 4, 2014. http://www. imeko.org/publications/wc-2006/PWC-2006-TC8-023u.pdf. 